ARTICLE

DOI: $10.1038 / s 41467-018-04689-5$

\title{
Non-fluorescent nanoscopic monitoring of a single trapped nanoparticle via nonlinear point sources
}

Seung Ju Yoon (1) 1, Jungmin Lee (10 1, Sangyoon Han', Chang-Kyu Kim², Chi Won Ahn (1) ${ }^{3}$, Myung-Ki Kim (1) ${ }^{4} \&$ Yong-Hee Lee ${ }^{1}$

Detection of single nanoparticles or molecules has often relied on fluorescent schemes. However, fluorescence detection approaches limit the range of investigable nanoparticles or molecules. Here, we propose and demonstrate a non-fluorescent nanoscopic trapping and monitoring platform that can trap a single sub-5-nm particle and monitor it with a pair of floating nonlinear point sources. The resonant photon funnelling into an extremely small volume of $\sim 5 \times 5 \times 7 \mathrm{~nm}^{3}$ through the three-dimensionally tapered 5-nm-gap plasmonic nanoantenna enables the trapping of a 4-nm CdSe/ZnS quantum dot with low intensity of a 1560-nm continuous-wave laser, and the pumping of 1560-nm femtosecond laser pulses creates strong background-free second-harmonic point illumination sources at the two vertices of the nanoantenna. Under the stable trapping conditions, intermittent but intense nonlinear optical spikes are observed on top of the second-harmonic signal plateau, which is identified as the 3.0- $\mathrm{Hz}$ Kramers hopping of the quantum dot trapped in the 5-nm gap.

\footnotetext{
${ }^{1}$ Department of Physics, Korea Advanced Institute of Science and Technology (KAIST), Daejeon 34141, South Korea. ${ }^{2}$ Department of Nano-Optical Engineering, Korea Polytechnic University, Siheung 15073, South Korea. ${ }^{3}$ KAIST, National NanoFab Center (NNFC), Daejeon 341401, South Korea. ${ }^{4}$ KU-KIST Graduate School of Converging Science and Technology, Korea University, Seoul 02841, South Korea. Correspondence and requests for materials should be addressed to M.-K.K. (email: rokmk@korea.ac.kr) or to Y.-H.L. (email: yhlee@kaist.ac.kr)
} 
ver the past decades, the majority of optical studies for single nanoparticles and molecules have been performed by the fluorescence detection scheme, where the spatial resolution is less than $10 \mathrm{~nm}$, breaking the diffraction limit. ${ }^{1-6}$ In the fluorescence detection scheme, the phosphor attached to the target substance acts as an isolated nano-light source, which allows the movement of the target substance to be traced in ultrahigh spatial resolution. However, an additional pre-process of attaching the fluorescent material is required, and the attachment sometimes produces unpredictable changes in the intrinsic properties of the target substance. The availability of alternative non-fluorescent detection would greatly expand the range of investigable nanoparticles or molecules beyond species that are emissive and photostable, thus offering new applications in the fields other than molecular biophysics and imaging. There have been numerous attempts to detect a single nanoparticle or a single molecule without relying on fluorescence detection schemes, such as high-precision surface-enhanced Raman spectroscopy (SERS), 7,8 cavity-enhanced detection methodology, ${ }^{9}$ interferometric scattering microscopy (iSCAT), ${ }^{10}$ centroid fitting methodology, ${ }^{11}$ and back-focal-plane interferometry. ${ }^{12}$

In recent years, plasmonically enhanced detection schemes using plasmonic nanoantennas, such as the circular double nanohole, ${ }^{13}$ nano-diabolo, ${ }^{14}$ nanorod, ${ }^{9,15}$ and bowtie ${ }^{16}$ plasmonic antennas, have actively been used for studies of single nanoparticles or molecules. The plasmonic nanoantenna can localize electromagnetic fields within a few nanometers, far beyond the diffraction limit, by utilizing surface-plasmon polaritons (SPPs), and thus strongly enhance the field intensity. Owing to the tight field concentration, the nanoantenna acts as a point-like illumination source that strongly increases the spatial resolution. ${ }^{17-19}$ Furthermore, the steep field gradient in the central region of the plasmonic nanoantenna generates an optical force strong enough to overcome the thermal Brownian fluctuations and to trap a single nanoparticle or a single molecule with low input power. ${ }^{20,21}$ In 2011, Pang et al. experimentally demonstrated the optical trapping of a single bovine serum albumin molecule with a 3.4-nm radius using a double nanoholes antenna. $^{22}$ They observed single-molecule trapping events using transmission signals. However, in most conventional nonfluorescent plamsonic antenna-based schemes, a single wavelength light is used for both trapping and monitoring. This makes it difficult to distinguish the monitoring light from the trapping light and to control the trapping and monitoring processes independently.
In this work, we report the label-free nanoscopic monitoring of a single trapped nanoparticle by illuminating it with a pair of nonlinear point-like sources built in a three-dimensionally tapered plasmonic nanoantenna. A two-beam resonant pump system comprising a $1560-\mathrm{nm}$ continuous-wave (CW) laser for trapping and a 1560-nm femtosecond (fs) laser for built-in second-harmonic ( $\mathrm{SH}$ ) illumination is employed for independent control of the trapping and monitoring processes. Resonant pumping into an extremely small volume of $5 \times 5 \times 7 \mathrm{~nm}^{3}$ through the tapered nanoantenna enables the low-power trapping of a nanoparticle and enhances nonlinear $\mathrm{SH}$ generation at the two vertices of the tapered nanoantenna. A pair of selfilluminating point-like nonlinear optical sources shines the quantum dot (QD) trapped in-between two potential wells formed in the nanoantenna. The Kramers hopping with a characteristic frequency of $3.0 \mathrm{~Hz}$ is identified by analysing the highcontrast nonlinear optical spikes.

\section{Results}

3D plasmonic nanoantenna. The proposed three-dimensional (3D) plasmonic nanoantenna platform is illustrated in Fig. 1a, in which a nanoscale air gap is introduced into a 100 -nm-thick Au metal layer on a $\mathrm{SiO}_{2}$ substrate. It can be observed that the two vertices are tapered along all three dimensions. The minimum nanogap is formed at the center and bottom of the antenna, where the electric field is strongly concentrated. ${ }^{23}$ In the finite-difference time-domain (FDTD) simulation, the mode volume and fieldintensity enhancement are calculated to be $6.1 \times 10^{-8} \lambda^{3}$ and $6.1 \times$ $10^{5}$, respectively (see Supplementary Note 1 ). The length, width, and central gap size of the antenna are 200,160, and $5.0 \mathrm{~nm}$, respectively. The resonant wavelength $\left(\lambda_{\omega}\right)$ of the antenna is $1560 \mathrm{~nm}$ in water. The incident laser (beam diameter $=2.0 \mu \mathrm{m}$ ) is pumped from the bottom. Figure $1 \mathrm{~b}$ shows the calculated $|E|^{2}$ profiles of the antenna mode in the $x y$ - and $y z$-plane. Two maximum $|E|^{2}$ points are notable at the apexes of the tapered nanoantenna. Here, the maximum electric fields are polarized perpendicular to the Au surface. ${ }^{24}$ Nonlinear SH signals $\left(\lambda_{2 \omega}=780 \mathrm{~nm}\right)$ are generated from the Au surface where the inversion symmetry is broken. ${ }^{25}$ In fact, the two maximum $|E|^{2}$ points at the tapered vertices act as a pair of floating nonlinear point sources. It is worth emphasizing that $\lambda_{2 \omega}$ is spectrally far enough from $\lambda_{\omega}$ such that the $\mathrm{SH}$ signal is background-free in principle. The $3 \mathrm{D}$ plasmonic nanoantenna is fabricated via proximal focused ion-milling (FIB) techniques on 100-nm-thick gold film

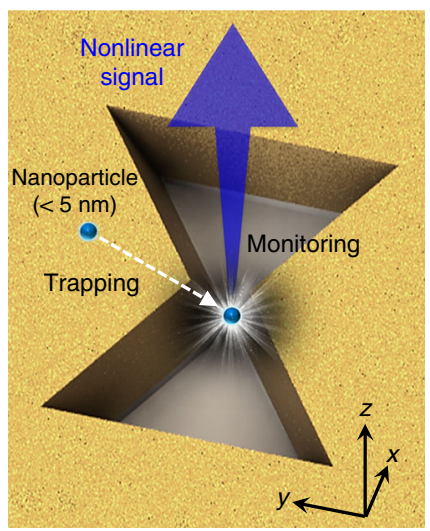

b

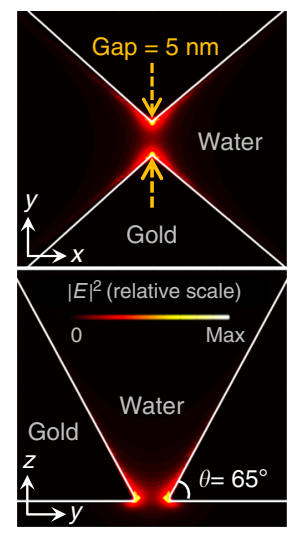

C

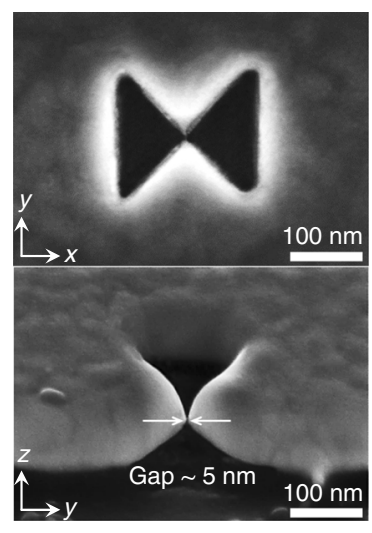

Fig. $13 \mathrm{D}$ plasmonic nanoantenna. a Schematic of the proposed 3D plasmonic nanoantenna. Squeezed photons in an extremely small volume $\left(V_{\mathrm{m}}=6.1 \times 10\right.$ $\left.-8 \lambda^{3}\right)$ of the 3D plasmonic nanoantenna enable the trapping of a sub-5-nm particle and create strong background-free second-harmonic signal. $\mathbf{b}|E|^{2}$ profiles of the antenna mode in the $x y$ - and $y z$-plane. The resonant wavelength of the antenna is $1560 \mathrm{~nm}$ in water. The $y$-polarized incident beam is pumped from the bottom to excite the resonant mode. c Scanning electron microscopy images of the fabricated 3D plasmonic nanoantenna. The centralgap size $(g)$ and the vertical taper angle are measured to be $\sim 5 \mathrm{~nm}$ and $\sim 65^{\circ}$, respectively 
a

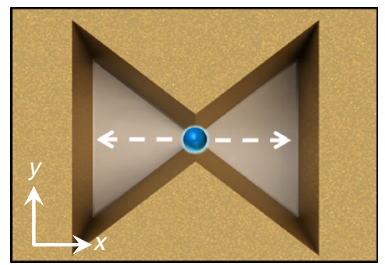

C

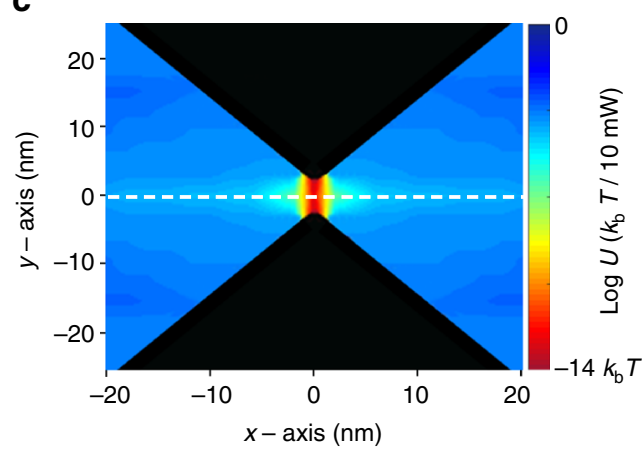

e

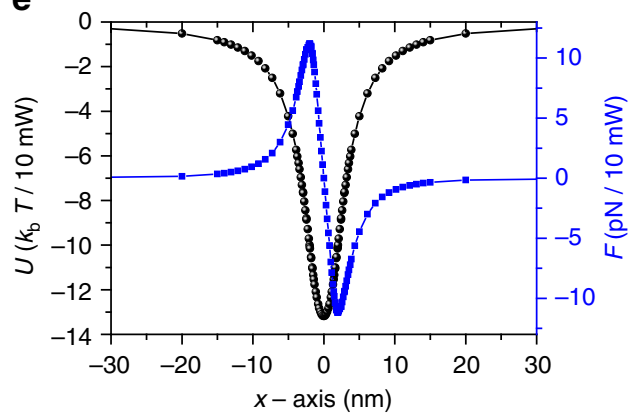

b

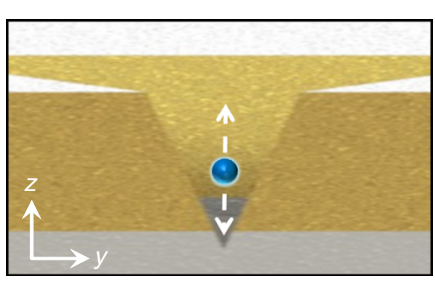

d

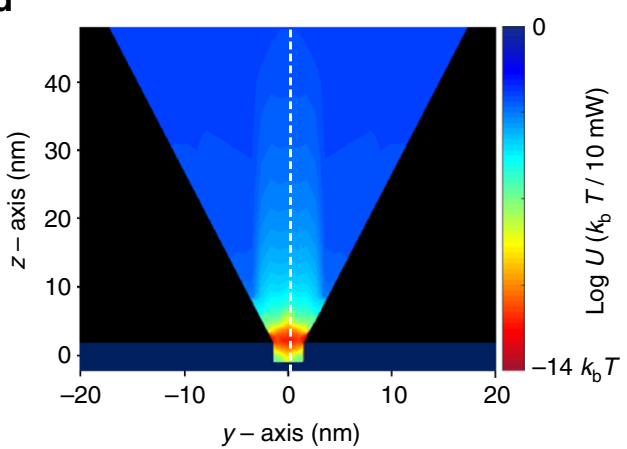

f

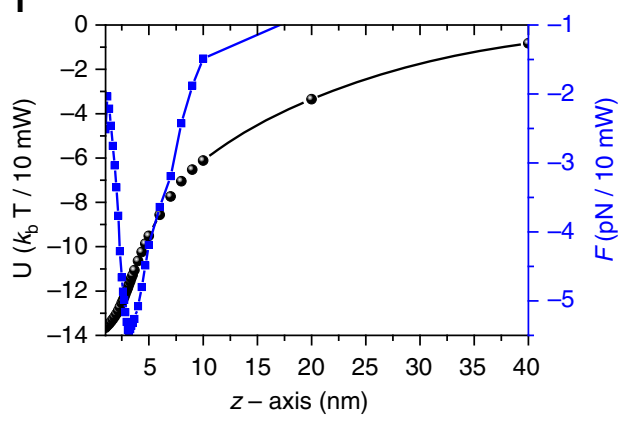

Fig. 2 Optical potentials in 3D plasmonic nanoantenna. a, b Cross-sectional views of the plasmonic nanoantenna in the $x y$-plane and $y z$-plane, respectively. c, $\mathbf{d}$ Calculated optical potentials (U) in logarithm scale for a quantum dot (QD) moving along the $x y$-plane and $y z$-plane, respectively. Here, the incident pump power, central-gap size $(\mathrm{g})$, and diameter of a QD are $10 \mathrm{~mW}, 5.0 \mathrm{~nm}$, and $4.4 \mathrm{~nm}$, respectively. e, $\mathbf{f}$ Optical potentials and the corresponding optical forces $(F)$ along the $x$ - and $z$-axis, respectively, near the center of the antenna

deposited on a quartz substrate, as shown in Fig. 1c (see Supplementary Note 2). Here, the central-gap size and the vertical taper angle are measured to be $\sim 5 \mathrm{~nm}$ and $\sim 65^{\circ}$, respectively.

Landscape of 3D optical potential well. The optical trapping potential applied to a 4.4-nm CdSe/ZnS QD is numerically obtained in the proximity of the $3 \mathrm{D}$ plasmonic nanoantenna, by solving the Maxwell stress tensors (MSTs) in the FDTD simulation (see Methods). The calculated potential maps along the $x y$-plane at $z=$ 0 and the $y z$-plane at $x=0$ for an incident pump power of $10 \mathrm{~mW}$ are shown in Fig. 2a, c and 2b, d, respectively. Here, the central-gap size is fixed at $5.0 \mathrm{~nm}$. The potential minimum is found at the center and bottom of the antenna, with a value of $-14 k_{\mathrm{b}} T$. Here, $k_{\mathrm{b}}$ is the Boltzmann constant, and $T$ is the temperature $(300 \mathrm{~K})$. This potential of $-14 k_{\mathrm{b}} T$ is deep enough to contain CdSe/ZnS QDs in the potential well. . $^{14,26-28}$ To obtain a better overview of the 3D potential landscape, we plot the potential and the corresponding optical force along the $x$ - and $z$-axis near the center of the antenna, as shown in Fig. 2e, f, respectively. The maximum restoring force is found to be $11.2 \mathrm{pN}$ when the QD is at $x= \pm 2.0 \mathrm{~nm}$. Along the $z$ direction, the optical force is largest at $z=3.2 \mathrm{~nm}$. For antennas with larger central gaps of 8 and $10 \mathrm{~nm}$, the corresponding potential depths are calculated to be $-9.3 k_{\mathrm{b}} T$ and $-2.0 k_{\mathrm{b}} T$, respectively (see Supplementary Note 3).

In the $y z$-plane potential profile, interesting features are observed. Figure $3 \mathrm{a}$ sketches an optical potential profile in the $y z$-plane for a 4.4-nm QD moving along the $y$-direction. The $y$ position is defined as the position of the QD projected onto the $y$ axis. We found two $14 k_{\mathrm{b}} T$-deep potential wells with a central barrier of height $0.6 k_{\mathrm{b}} T$. Note that two potential minima $(y=$ $\pm 1.5 \mathrm{~nm}$ ) are located near the plasmonic hot points close to the apexes of the nanoantenna in Fig. 1b. The height $0.6 k_{\mathrm{b}} T$ of the central barrier is not large enough to stably hold a thermal QD in one of the two wells. Therefore, the QD can hop over the central barrier between two potential wells whose height tends to increase with the pump power (see Supplementary Note 4).

The electric-field profile also changes as the QD moves in the potential well, as shown in Fig. 3b. The solid blue dot in Fig. 3a represents the $|E|^{2}$ enhancement factor with respect to the $|E|^{2}$ of incident light. When the QD is placed at the bottom of the potential well (P1 position in Fig. 3, $y= \pm 1.5 \mathrm{~nm}$ ), the $|E|^{2}$ enhancement is calculated to be $1.1 \times 10^{6}$, which is 1.8 times larger than that obtained in the absence of a QD. When the QD is placed exactly on top of the potential barrier ( $\mathrm{P} 2$ position in Fig. 3), the $|E|^{2}$ enhancement is maximized to be $2.0 \times 10^{6}$, which is 1.8 times larger than that of the QD at P1. This is because the low-refractive index region in the 5.0-nm gap is minimized when the $\mathrm{QD}$ is at $\mathrm{P} 2$, as shown in Fig. 3b. What we measure in the experiment is the nonlinear $\mathrm{SH}$ signals proportional to the value of $|E|^{4}$ at the $\mathrm{Au}$ surface. $^{22,29,30}$ 
a

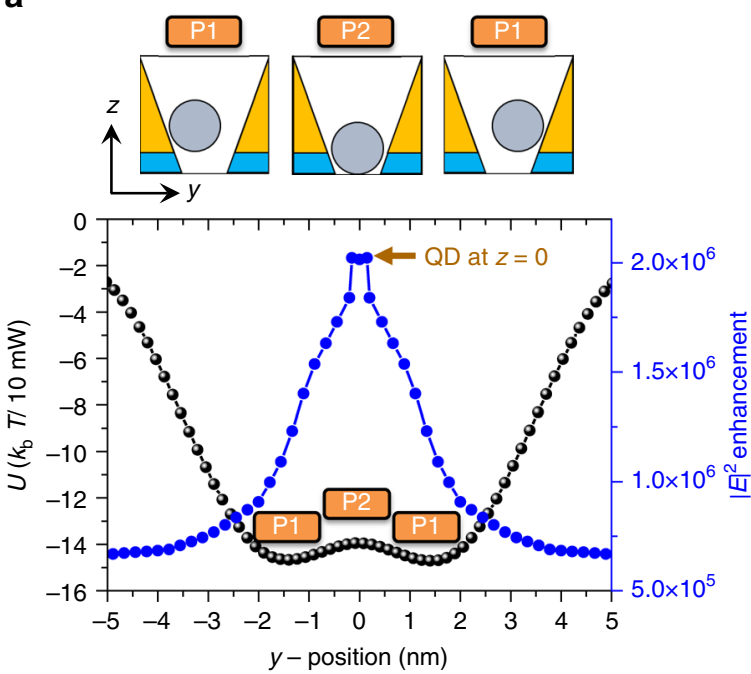

b

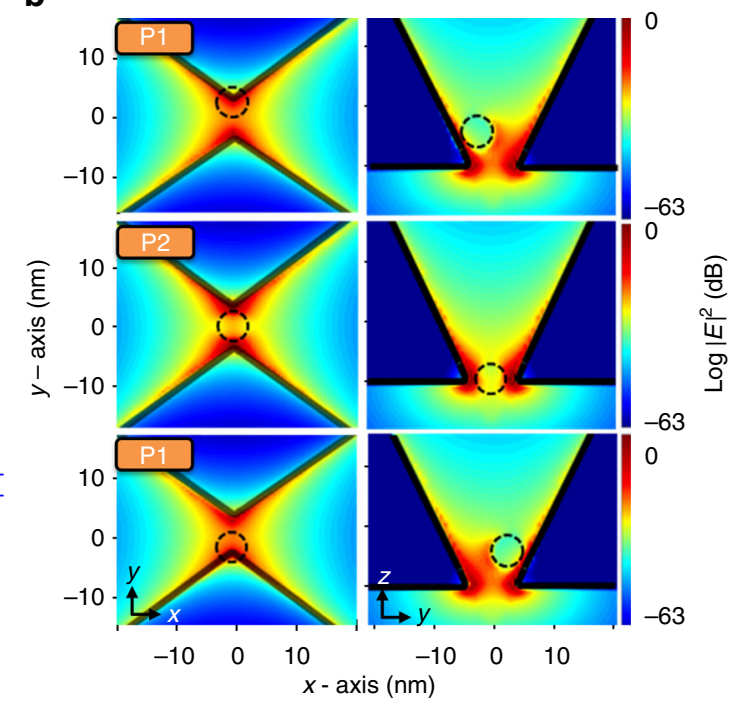

Fig. 3 Double potential well in 3D plasmonic nanoantenna. a Optical potential $(U)$ and $|E|^{2}$ enhancement factor as a 4.4-nm quantum dot (QD) moves along the $y$-direction. A double potential well with two $14 k_{\mathrm{b}} T$-deep wells and a central barrier of height $0.6 k_{\mathrm{b}} T$ is observed. Here, the $y$-position is defined as the projected position on the $y$-axis and the incident beam power is $10 \mathrm{~mW}$. $\mathbf{b}|E|^{2}$ profiles in $x y$-planes and $y z$-planes when a QD is located at the positions of the potential well (P1) and the central barrier (P2). When a QD is placed at $P 2$, the $|E|^{2}$ enhancement is maximized to be $2.0 \times 10^{6}$, which is 1.8 times larger than that of the QD at P1. This is because the low-refractive index region in the 5.0-nm gap is minimized when a QD is at P2

a

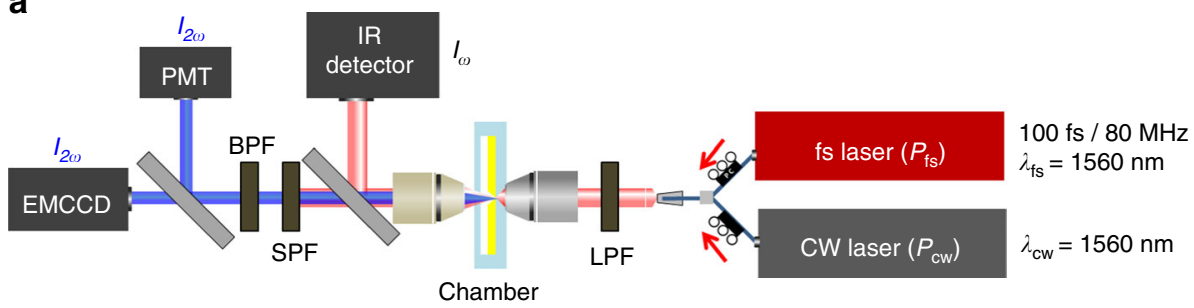

b

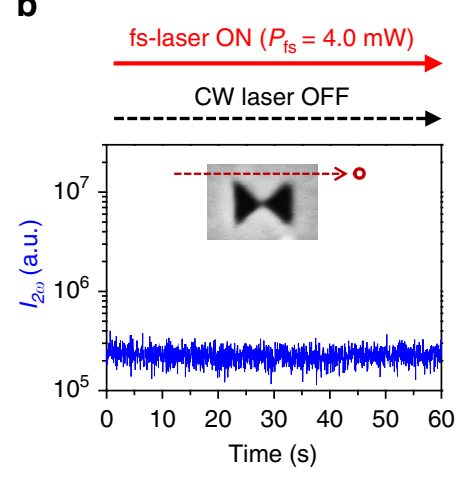

C

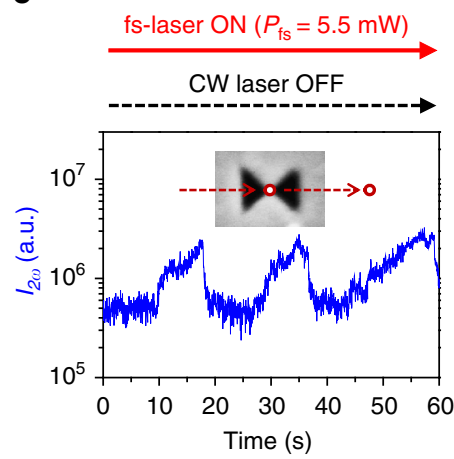

d

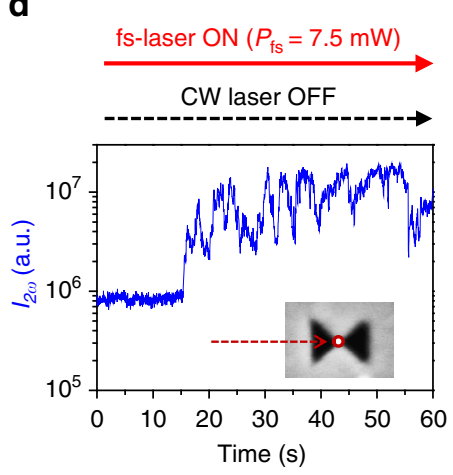

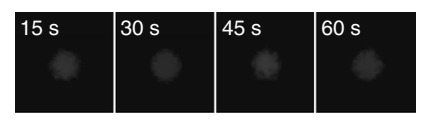
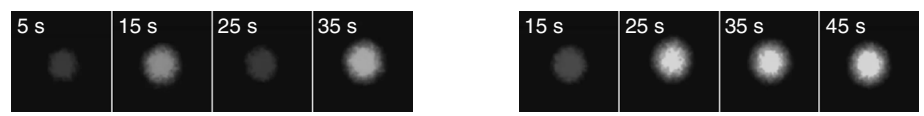

Fig. 4 Experimental setup and monitoring of second-harmonic signals. a Two-laser scheme for simultaneous trapping and monitoring. A femtosecond (fs) pulse laser and a continuous-wave (CW) laser are independently used for monitoring and trapping, respectively. The two lasers are combined with a 50:50 coupler and the short wavelengths of the laser beam are filtered with a long-pass filter (LPF). The transmitted fundamental wave signal $\left(I_{\omega}\right)$ is monitored by an infrared photodetector, and the short-wavelength second-harmonic (SH) signal ( $\left.I_{2 \omega}\right)$ filtered by a short-pass filter (SPF) and a band-pass filter (BPF) is monitored by an electron multiplying charge-coupled device (EMCCD) and a photomultiplier tube (PMT). b-d Time traces of SH intensities ( $I_{2 \omega}$ ) with different fs-laser powers $\left(P_{\mathrm{fs}}=4.0,5.5\right.$, and $\left.7.5 \mathrm{~mW}\right)$ in the absence of the $\mathrm{CW}$ trapping laser. When $P_{\mathrm{fs}}=4.0 \mathrm{~mW}$, a stable nonlinear monitoring signal is observed 

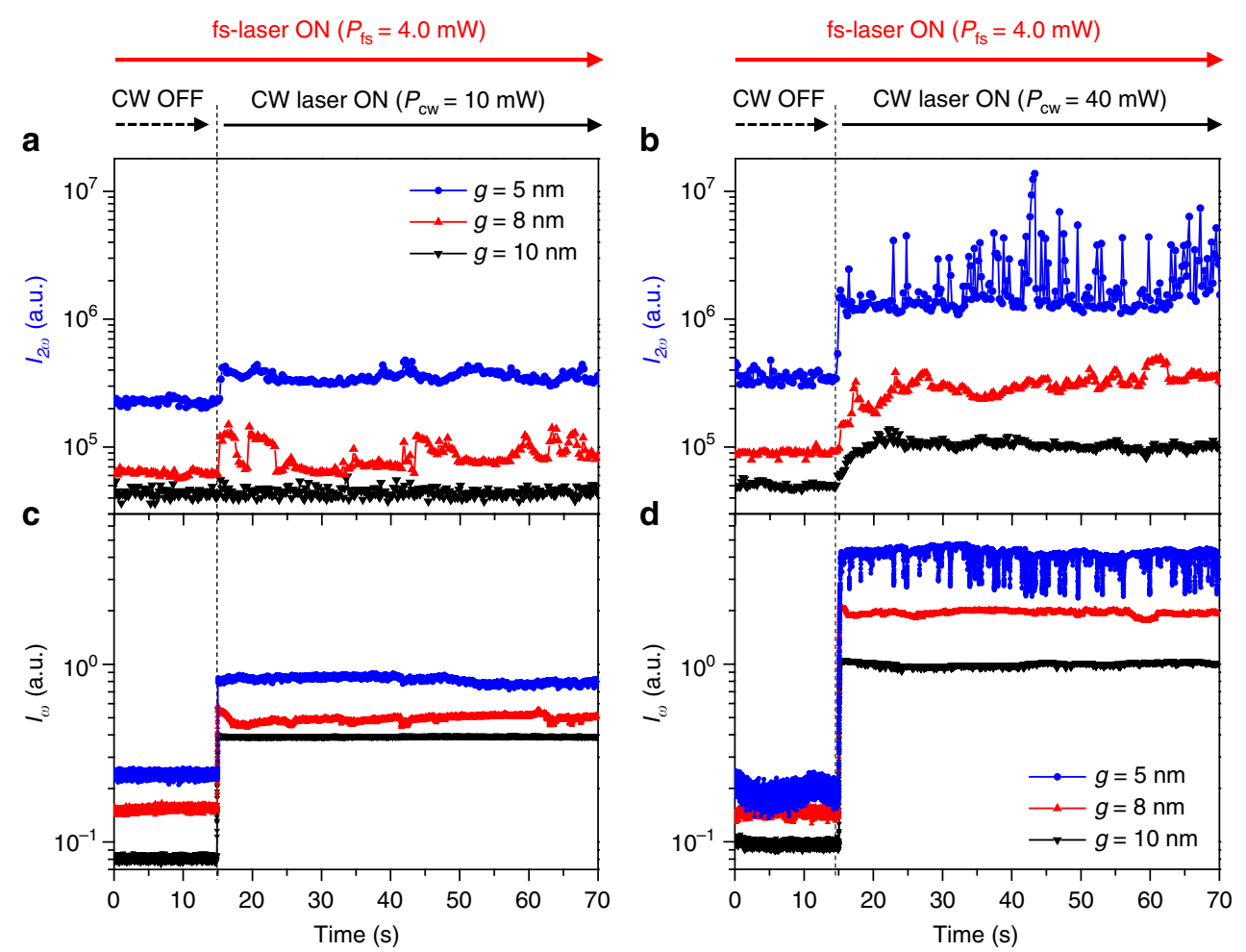

Fig. 5 Simultaneous trapping and monitoring. $\mathbf{a}, \mathbf{b}$ Time traces of second harmonic intensities $\left(I_{2 \omega}\right)$ and $\mathbf{c}, \mathbf{d}$ transmitted fundamental wave intensities $\left(I_{\omega}\right)$ with different $\mathrm{CW}$ laser powers of 10 and $40 \mathrm{~mW}$, respectively, for three different nanoantennas with gap sizes $(\mathrm{g})$ of 5,8 , and $10 \mathrm{~nm}$. The $\mathrm{CW}$ laser is turned on at $t=15 \mathrm{~s}$, and the 4.0-mW fs-laser remains on. The integration times for $I_{\omega}$ and $I_{2 \omega}$ detection are 2 and $200 \mathrm{~ms}$, respectively. Looking at the trapped moment $(t=15 \mathrm{~s})$ of the quantum dot (QD), monitoring the $I_{\omega}$ signal makes it difficult to distinguish whether the QD is trapped, due to a sudden increase in the $I_{\omega}$ signal. On the other hand, the $I_{2 \omega}$ signal clearly tells us whether the QD is trapped or not and even shows the dynamics of the trapped QD

Experiment. Armed with the theoretical study, we test our nonfluorescent trapping platform in a confocal optical setup [Fig. 4a, see Methods]. Two lasers-a fs pulse laser (100-fs pulses at 80 $\mathrm{MHz}$ ) and a CW laser-are tuned to $1,560 \mathrm{~nm}$ for the resonant excitation of nanoantennas, for monitoring and trapping, respectively. The beam diameter focused through a $60 \times$ objective lens (numerical aperture $=0.65$ ) is measured to be $2.0 \mu \mathrm{m}$, and the two lasers are polarized along the $y$-direction. The transmitted and reflected light is collected by photodetectors and an electronmultiplying charged-coupled device (EMCCD). CdSe/ZnS QDs (diameter $\sim 4.0 \mathrm{~nm}, \lambda_{\text {emission }} \sim 620 \mathrm{~nm}$ ) are dissolved in water with concentration of $2.0 \times 10^{-6} \mathrm{M}$. Then they are injected into a chamber equipped with $3 \mathrm{D}$ nanoantennas. Note that the wavelengths of both pump beam $\left(\lambda_{\omega}=1.56 \mu \mathrm{m}\right)$ and SH signals $\left(\lambda_{2 \omega}\right.$ $=0.78 \mu \mathrm{m})$ are transparent to CdSe/ZnS QDs of interest.

First, the range of the fs-laser power $P_{\mathrm{fs}}$ suitable for nonlinear $\mathrm{SH}$ generation is investigated, in the absence of the $\mathrm{CW}$ trapping laser. With a low fs-laser power of $4.0 \mathrm{~mW}$ (intensity $I_{\mathrm{fs}}=P_{\mathrm{fs}} /$ $A_{\text {beam }}=0.12 \mathrm{MW} \mathrm{cm}^{-2}$, where $A_{\text {beam }}$ is the area of the focused beam), stable nonlinear light $I_{2 \omega}$ is observed, as shown in Fig. $4 \mathrm{~b}$. We take this baseline $I_{2 \omega}$ as the background noise level at the monitoring wavelength of $\lambda_{2 \omega}$. Here, the quietness of the baseline indicates the non-trapping of QD. When $P_{\mathrm{fs}}$ is increased to 5.5 $\mathrm{mW}\left(I_{\mathrm{fs}}=0.18 \mathrm{MW} \mathrm{cm}^{-2}\right)$, as shown in Fig. 4c, the QD becomes trapped lightly and then escapes shortly in a random fashion. If $P_{\mathrm{fs}}$ is further increased to $7.5 \mathrm{~mW}\left(I_{\mathrm{fs}}=0.24 \mathrm{MW} \mathrm{cm}^{-2}\right)$, the QD remains trapped, as shown in Fig. $4 \mathrm{~d}$. In all the subsequent experiments, the fs-laser power level is set to $4.0 \mathrm{~mW}$ to minimize the baseline $I_{2 \omega}$.

The fs-laser and trapping CW laser are turned on at $t=0$ and $15 \mathrm{~s}$, respectively. In the regions of Fig. $5 \mathrm{a}, \mathrm{b}$ where $t<15 \mathrm{~s}$, we observe a clear dependence of the nonlinear background level on the gap size $g$, which is ascribed to the varying field strength. ${ }^{23}$ The relative heights of the background baselines are measured to be $5.1,1.5$, and 1.0 , for $g=5,8$, and $10 \mathrm{~nm}$, respectively. The corresponding relative transmittances of the fundamental wave $\left(\lambda_{\omega}\right)$ are measured to be $2.9,1.9$, and 1.0, respectively, as shown in Fig. $5 c$, d. We also confirm that the pump CW laser produces a negligible $\mathrm{SH}$ background signal (see Supplementary Note 5).

When the CW laser is turned on $(t=15 \mathrm{~s})$ in addition to the 4$\mathrm{mW}$ fs-laser, the transmittance of the trapping $\mathrm{CW}$ laser $I_{\omega}$ at $1560 \mathrm{~nm}$ and the $\mathrm{SH}$ monitoring signal $I_{2 \omega}$ at $780 \mathrm{~nm}$ exhibit rapid and dramatic changes. In our nanoscopy platform, two floating $\mathrm{SH}$ point sources at the vertices of nanoantenna automatically illuminate the QD once it is trapped between them. Because the $\mathrm{SH}$ signal has a quadratic dependence on the intensity, the $I_{2 \omega}$ SH signal is expected to be a sensitive function of the QD position in the optical potential. That is, the signature of ultrafine motion of the QD in the 5-nm gap is recorded faithfully in the $I_{2 \omega}$ signal, with a high signal-to-noise ratio. Looking at the trapped moment $(t=15 \mathrm{~s})$ of the QD, monitoring the $I_{\omega}$ signal makes it difficult to distinguish whether the QD is trapped, due to a sudden increase in the $I_{\omega}$ signal when the trapping CW laser is turned on. On the other hand, the $I_{2 \omega}$ signal clearly tells us whether the QD is trapped or not and even shows the dynamics of the trapped QD. The CW trapping laser power of $10 \mathrm{~mW}\left(I_{\mathrm{cW}}=0.32 \mathrm{MW} \mathrm{cm}^{-2}\right)$ is found to be sufficient to trap a QD stably for the nanoantenna with a small gap of $g=5 \mathrm{~nm}$ but insufficient for antennas with a larger gap, as shown in Fig. 5.

When the trapping laser power $P_{\mathrm{cw}}$ is increased to $40 \mathrm{~mW}\left(I_{\mathrm{cw}}\right.$ $=1.27 \mathrm{MW} \mathrm{cm}^{-2}$ ), stable trapping is observed for all three antenna structures with $g=5,8$, and $10 \mathrm{~nm}$. It is confirmed that a QD can be trapped in the antenna for more than $2 \mathrm{~h}$. The trapping time, which is the time required for a $\mathrm{QD}$ to reach a 
a

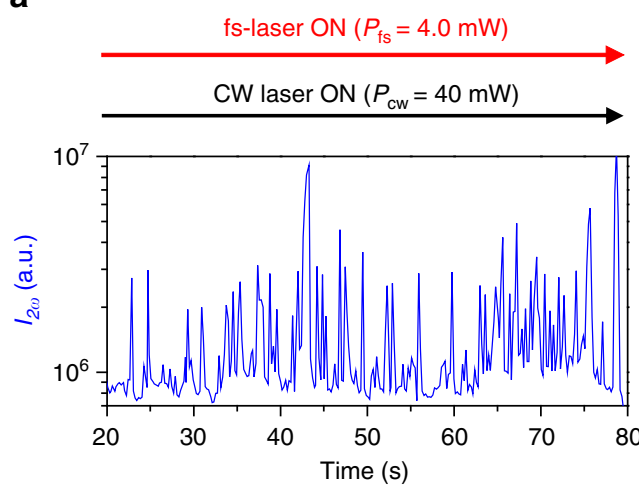

c

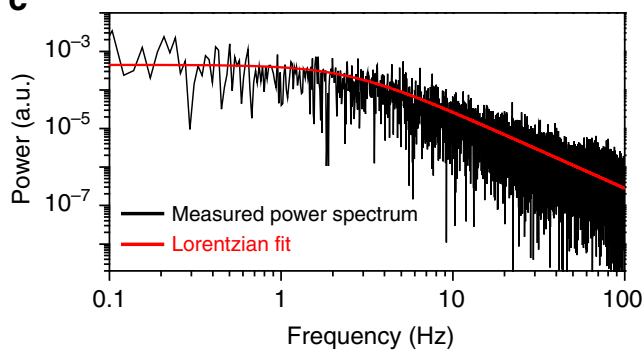

b

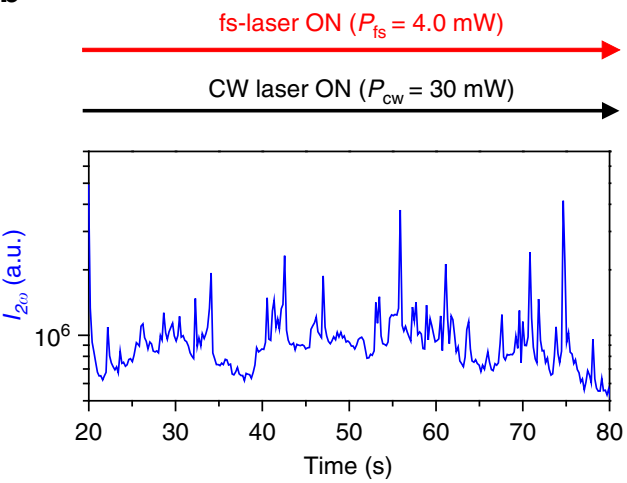

d

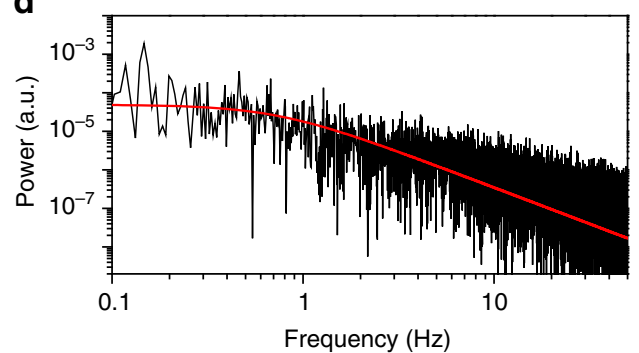

Fig. 6 Kramers hopping. a, b Measured second harmonic $(\mathrm{SH})$ spike signals $\left(I_{2 \omega}\right)$ with trapping $\mathrm{CW}$ laser powers $P_{\mathrm{cw}}$ of 40 and $30 \mathrm{~mW}$, respectively, where the 4.0-mW fs-laser is on. These signals are estimated to be generated by the low frequency Kramers hopping associated with the double potential well formed near the center of the nanoantenna. c, d Power spectra (black lines) of the measured SH signals and the Lorentzian fitting curves (red lines). When $P_{\mathrm{cW}}$ is 40 and $30 \mathrm{~mW}$, the roll-off frequency is measured as 3.0 and $0.5 \mathrm{~Hz}$, respectively

stable trapped state, shows a noticeable gap-size dependence. It is measured as $0.5,2.8$, and $6.4 \mathrm{~s}$ for $g=5,8$, and $10 \mathrm{~nm}$, respectively. With a $4-\mathrm{mW}$ fs-laser, the average $\mathrm{SH}$ intensities normalized to the nonlinear background level are measured to be 5.6, 3.3, and 2.1, for $g=5,8$, and $10 \mathrm{~nm}$, respectively. Once the CW laser is turned off, the QD escapes the antenna immediately (see Supplementary Note 5).

\section{Discussion}

A series of intense $\mathrm{SH}$ spikes are observed from a trapped 4-nm $\mathrm{CdSe} / \mathrm{ZnS} \mathrm{QD}$ when the 3D tapered nanoantenna is resonantly pumped at a wavelength of $1560 \mathrm{~nm}$ with a $40-\mathrm{mW}$ CW laser and a 4 - $\mathrm{mW}$ fs-laser, as shown in Fig. 5b. Additionally, in the transmitted fundamental signal $\left(I_{\omega}\right)$, similar negative spikes are simultaneously observed mainly due to the translational movement of the trapped QD, as shown in Fig. 5d (see Supplementary Note 5). We attribute this phenomenon to the Kramers hopping associated with the double potential well formed near the two hot plasmonic points discussed previously, as shown in Fig. 3. The low-frequency characteristics of the $\mathrm{SH}$ spikes supports our interpretation. ${ }^{19}$ That is, the QD hops back and forth between two shallow potential wells (P1 positions in Fig. 3) randomly at a low frequency. It generates a strong $\mathrm{SH}$ signal spike at the instant that the $\mathrm{QD}$ passes by the $\mathrm{P} 2$ position in Fig. 3. When the QD is located at P2, the field intensity at the antenna gap is calculated to be 1.8 times larger than that at the bottom of the well (P1), as shown in Fig. 3. Dielectric loading of the nano-metallic gap with a trapped QD is found to enhance the electric field and surface current density responsible for the $\mathrm{SH}$ generation. The $\mathrm{SH}$ signal intensity with a QD at P2 is expected to increase by a factor of $1.8^{2}$ $(=3.24)$ with respect to that with a QD at P1. This ratio agrees with the average intensity increment (3.26 times) of the intermittent SH spikes shown in Fig. 6a. The hopping rate is analysed by using the power spectrum density method, as shown in Fig. 6c.
The roll-off frequency (corner frequency) of the Kramers hopping is estimated to be $3.0 \mathrm{~Hz}$. In additional experiments, we found that the roll-off frequency decreases with decreasing $P_{\mathrm{cw}}$ (see Supplementary Note 6). This is understandable considering that the height of the potential barrier decreases with decreasing $P_{\mathrm{cw}}$. For example, when $P_{\mathrm{cw}}=30 \mathrm{~mW}$, the roll-off frequency is measured as $0.5 \mathrm{~Hz}$, as shown in Fig. $6 \mathrm{~b}$, d. This is the unambiguous observation that a single nanoparticle trapped in the plasmonic nanoantenna exhibits Kramers hopping.

In summary, we have demonstrated the non-fluorescent nanoscopic monitoring of a single trapped nanoparticle by illuminating it with a pair of floating nonlinear point sources built in a plasmonic nanoantenna. A two-beam resonant pump system comprising a 1560-nm CW laser for trapping and a fs-laser for built-in SH illumination is employed for independent control of the trapping and monitoring processes. The resonant pumping into an extremely small mode volume of $5 \times 5 \times 7 \mathrm{~nm}^{3}$ enables the trapping and monitoring of a $4-\mathrm{nm} \mathrm{CdSe} / \mathrm{ZnS}$ QD with low optical intensity less than $0.4 \mathrm{MW} \mathrm{cm}^{-2}$. Intense nonlinear optical spikes are observed when a $4-\mathrm{nm}$ CdSe/ZnS QD crosses the potential barrier in the middle of a symmetric double potential well formed between two apexes of the threedimensionally tapered plasmonic nanoantenna with 5-nm gap. The Kramers hopping of the $3.0-\mathrm{Hz}$ characteristic frequency is identified by analysing the high-contrast nonlinear optical spikes. The label-free and high-contrast characteristics of the proposed scheme are believed to be advantageous for future quantum and bio-optical research.

\section{Methods}

Optical potential and force calculations. The electromagnetic force applied to a particle by harmonic fields can be calculated using the Maxwell stress tensor (MST). We calculate the electromagnetic field around the particle in an FDTD simulation and apply the MST method. The simulations are conducted numerically 
using a commercially available FDTD software package (Lumerical Solutions, Inc.; https://www.lumerical.com/).

The Lorentz force per unit volume is

$$
\mathbf{f}=\rho \mathbf{E}+\mathbf{J} \times \mathbf{E},
$$

where $\rho$ is the total charge per unit volume, and $\mathbf{J}$ is the total current density.

According to Maxwell's equations,

$$
\rho=\varepsilon \nabla \cdot \mathbf{E}, \mathbf{J}=\frac{1}{\mu} \nabla \times \mathbf{B}-\varepsilon \frac{\partial \mathbf{E}}{\partial t},
$$

and according to the product rule,

$$
\frac{\partial}{\partial t}(\mathbf{E} \times \mathbf{B})=\frac{\partial \mathbf{E}}{\partial t} \times \mathbf{B}+\mathbf{E} \times \frac{\partial \mathbf{B}}{\partial t} .
$$

Eq. (1) then becomes

$$
\mathbf{f}=\varepsilon[(\nabla \cdot \mathbf{E}) \mathbf{E}-\mathbf{E} \times(\nabla \times \mathbf{E})]+\frac{1}{\mu}[(\nabla \cdot \mathbf{B}) \mathbf{B}-\mathbf{B} \times(\nabla \times \mathbf{B})]-\varepsilon \frac{\partial}{\partial t}(\mathbf{E} \times \mathbf{B}) .
$$

After eliminating the curls, using the vector calculus identity, we obtain

$$
\nabla(\mathbf{A} \cdot \mathbf{B})=(\mathbf{A} \cdot \nabla) \mathbf{B}+(\mathbf{B} \cdot \nabla) \mathbf{A}+\mathbf{A} \times(\nabla \times \mathbf{B})+\mathbf{B} \times(\nabla \times \mathbf{A}) .
$$

With the Poynting vector $\mathbf{S}=\frac{1}{\mu} \mathbf{E} \times \mathbf{B}$, Eq. (4) is expressed as

$$
\begin{aligned}
\mathbf{f} & =\varepsilon\left[(\nabla \cdot \mathbf{E}) \mathbf{E}-(\mathbf{E} \cdot \nabla) \mathbf{E}-\frac{1}{2} \nabla E^{2}\right] \\
& +\frac{1}{\mu}\left[(\nabla \cdot \mathbf{B}) \mathbf{B}-(\mathbf{B} \cdot \nabla) \mathbf{B}-\frac{1}{2} \nabla B^{2}\right]-\varepsilon \mu \frac{\partial \mathbf{S}}{\partial t} .
\end{aligned}
$$

Now, the MST $\bar{T}$ is a $3 \times 3$ matrix with components defined by

$$
\bar{T}_{i j}=\varepsilon\left(\mathrm{E}_{i} \mathrm{E}_{j}-\frac{1}{2} \delta_{i j} E^{2}\right)+\frac{1}{\mu}\left(\mathrm{B}_{i} \mathrm{~B}_{j}-\frac{1}{2} \delta_{i j} B^{2}\right) .
$$

The last term in Eq. (6) is eliminated via time averaging. As a result, the timeaveraged force is simplified as

$$
\langle\mathbf{f}\rangle_{\text {time-averaged }}=\langle\nabla \cdot \mathbf{T}\rangle_{\text {time-averaged }} .
$$

Thus, the total force on the particle is obtained as the volume integral of the particle.

Using the relationship between the force and the potential, we obtain

$$
\mathrm{U}=-\int_{\text {ref }}^{r} \overrightarrow{\mathbf{F}} \cdot \mathrm{d} \overrightarrow{\mathbf{r}} \text {. }
$$

Thus, the optical potential is calculated. The potential at infinity is considered to be zero.

In the FDTD simulations, we compute the electromagnetic field numerically when the $4.4 \mathrm{~nm}$ size particle is in each position in the cavity. The grid size of FDTD calculation is $0.5 \mathrm{~nm} \times 0.5 \mathrm{~nm} \times 0.5 \mathrm{~nm}$. The nanoantenna dimensions are considered to be $200 \times 160 \times 100 \mathrm{~nm}$ in length, width, and thickness, respectively, and the $3 \mathrm{D}$ taper angle is $65^{\circ}$.

Optical measurements. We use the confocal optical setup and control the trapping event time by separating the probe laser and trapping laser, as shown in Fig. 4a. A femtosecond pulse laser (Toptica FemtoFiber pro NIR, $\lambda=1.56 \mu \mathrm{m}, 100-$ fs pulse, $80 \mathrm{MHz}$ ) with a peak power of approximately $0.5 \mathrm{~kW}$ is employed as a probe laser for generating the SH signal and illuminates the cavity with an incident power of $0.12 \mathrm{MW} \mathrm{cm}^{-2}$ during the optical trapping. A CW laser (Thorlab, Product No. FPL1009S, $\lambda=1.56 \mu \mathrm{m}$ ) is additionally introduced into the cavity with an incident power from 0.32 up to $1.27 \mathrm{MW} \mathrm{cm}{ }^{-2}$ and independently controls trapping events as a trapping laser. The two beams are combined through a 50:50 beam coupler and focused onto the sample through the microscope objective (60×, $0.65 \mathrm{NA})$ with transverse direction polarization ( $y$-axis in Fig. 1). The transmitted fundamental beam is detected by an infrared photodiode (Femtowatt InGaAs Photoreceiver, Newport, model:2153). Simultaneously, the SH signal is detected by the visible EMCCD (Andor technology, iXon3 897) and visible photomultiplier tube (Princeton Instruments, PD-471) after passing through both the short-pass filter $\left(\mathrm{SPF}, \lambda_{\text {cut-off }}=1300 \mathrm{~nm}\right)$ and the bandpass filter $\left(\mathrm{BPF}, \lambda_{\text {center }}=780 \pm 10 \mathrm{~nm}\right)$. The time traces of the $\mathrm{SH}$ and fundamental signal are recorded simultaneously in real time. Prior to the optical trapping experiments, the nanoantenna is integrated in the microfluidic chamber with QDs dissolved in water with concentration of $2 \times 10$

${ }^{-6} \mathrm{M}$. The QD (Nanosquare, Product No. C01-SH01-AC-100620, standard emission peak $=620 \mathrm{~nm}$ ) is CdSe/ZnS (core/shell type) and $4.4 \mathrm{~nm}$ in diameter, as specified by the manufacturer.

Data availability. The data sets within the article and Supplementary Information of the current study are available from the authors upon request.
Received: 20 November 2017 Accepted: 16 May 2018

Published online: 07 June 2018

\section{References}

1. Adams, S. R., Harootunian, A. T., Buechler, Y. J., Taylor, S. S. \& Tsien, R. Y. Fluorescence ratio imaging of cyclic AMP in single cells. Nature 349, 694-697 (1991).

2. Lakowicz, J. R. \& Szmacinski, H. Fluorescence lifetime-based sensing of pH, $\mathrm{Ca} 2+, \mathrm{K}+$ and glucose. Sens. Actuators B 11, 1-3 (1993).

3. Altschuh, D., Oncul, S. \& Demchenko, A. P. Fluorescence sensing of intermolecular interactions and development of direct molecular biosensors. J. Mol. Recognit. 19, 459-477 (2006).

4. Tagit, O. \& Hildebrandt, N. Fluorescence sensing of circulating diagnostic biomarkers using molecular probes and nanoparticles. ACS Sens. 2, 31-45 (2017).

5. Lakowicz. et al. Novel methods for fluorescence sensing. In Proc. SPIE, Advances in Fluorescence Sensing Technology IV 3602 https://doi.org/10.1117/ 12.347525 (1999).

6. Lippitsch, M., Draxler, S. \& Kieslinger, D. Luminescence lifetime-based sensing: new materials, new devices. Sens. Actuators B 38, 1-3 (1997).

7. Yampolsky, S. et al. Seeing a single molecule vibrate through time-resolved coherent anti-Stokes Raman scattering. Nat. Photon 8, 650-656 (2014).

8. Harmsen, S. et al. Surface-enhanced resonance Raman scattering nanostars for high-precision cancer imaging. Sci. Transl. Med 7, 271 (2015).

9. Zijlstra, P., Paulo, P. M. R. \& Orrit, M. Optical detection of single nonabsorbing molecules using the surface plasmon resonance of a gold nanorod. Nat. Nanotechnol. 7, 379-382 (2012).

10. Cole, D., Young, G., Weigel, A., Sebesta, A. \& Kukura, P. Label-free singlemolecule imaging with numerical-aperture-shaped interferometric scattering microscopy. ACS Photonics 4, 211-216 (2017).

11. Brzobohatý, O. et al. Three-dimensional optical trapping of a plasmonic nanoparticle using low numerical aperture optical tweezers. Sci. Rep. 5, 8106 (2015).

12. Gittes, F. \& Schmidt, C. F. Interference model for back-focal-plane displacement detection in optical tweezers. Opt. Lett. 23, 7-9 (1998).

13. Kotnala, A. \& Gordon, R. Quantification of high-efficiency trapping of nanoparticles in a double nanohole optical tweezer. Nano Lett. 14, 853-856 (2014).

14. Kang, J.-H. et al. Low-power nano-optical vortex trapping via plasmonic diabolo nanoantennas. Nat. Commun. 2, 582 (2011).

15. Zhang, W., Huang, L., Santschi, C. \& Martin, O. J. F. Trapping and sensing 10 $\mathrm{nm}$ metal nanoparticles using plasmonic dipole antennas. Nano Lett. 10, 1006-1011 (2010)

16. Berthelot, J. et al. Three-dimensional manipulation with scanning near-field optical nanotweezers. Nat. Nanotechnol. 9, 295-299 (2014).

17. Roxworthy, B. J. \& Toussaint, K. C. Jr. Femtosecond-pulsed plasmonic nanotweezer. Sci. Rep. 2, 660 (2012).

18. Kim, S. et al. High-harmonic generation by resonant plasmon field enhancement. Nature 453, 757-760 (2008).

19. Nicoli, F., Verschueren, D., Klein, M., Dekker, C. \& Jonsson, M. P. DNA translocations through solid-state plasmonic nanopores. Nano Lett. 14 6917-6925 (2014)

20. Marin, B. C. et al. Plasmon-enhanced two-photon absorption in photoluminescent semiconductor nanocrystals. ACS Photonics 3, 526-531 (2016).

21. Zehtabi-Oskuie, A. et al. Double nanohole optical trapping: dynamics and protein-antibody co-trapping. Lab. Chip. 13, 2563-2568 (2013).

22. Pang, Y. \& Gordon, R. Optical trapping of a single protein. Nano Lett. 12 402-406 (2012).

23. Kim, M.-K. et al. Squeezing photons into a point-like space. Nano Lett. 15, 4102-4107 (2015)

24. Pitarke, J. M., Silkin, V. M., Chulkov, E. V. \& Echenique, P. M. Theory of surface plasmons and surface-plasmon polaritons. Rep. Prog. Phys. 70, 1 (2007).

25. Terhune, R. W., Maker, P. D. \& Savage, C. M. Optical harmonic generation in calcite. Phys. Rev. Lett. 8, 404 (1962).

26. Ashkin, A., Dziedzic, J. M., Bjorkholm, J. E. \& Chu, S. Observation of a singlebeam gradient force optical trap for dielectric particles. Opt. Lett. 11, 288-290 (1986).

27. Righini, M. et al. Nano-optical trapping of Rayleigh particles and Escherichia coli bacteria with resonant optical antennas. Nano Lett. 9, 3387-3391 (2009)

28. Ploschner, M., Mazilu, M., Krauss, T. F. \& Dholakia, K. Optical forces near a nanoantenna. J. Nanophotonics. 4, 041570 (2010).

29. Ng, J. C., Herman, P. R. \& Qian, L. Second harmonic generation via femtosecond laser fabrication of poled, quasi-phase-matched waveguides in fused silica. Opt. Lett. 42, 2 (2017). 
30. Kuo, P. S., Bravo-Abad, J. \& Solomon, G. S. Second-harmonic generation using quasi-phase matching in a GaAs whispering-gallery-mode microcavity. Nat. Commun. 5, 3109 (2014).

\section{Acknowledgements}

This work was supported by the National Research Foundation of Korea (NRF) grants (NRF-2017M3C1A3013923 and NRF-2015K1A4A3047100). M.-K. Kim acknowledges support received from the NRF grant (NRF-2017R1D1A1B03036010), the KIST Institutional Program (2E26680-16-P024), and the KU-KIST School Project. S. Han acknowledges support received from the NRF grant (NRF-2017R1D1A1B03036289) and the Ministry of Science and ICT of Korea grant (Global Center for Open Research with Enterprise).

\section{Author contributions}

S.Y., M.K., and Y.L. conceived the idea and designed the experiments. S.Y. fabricated the samples, and S.Y., J.L., M.K., and Y.L. performed the experiments and analysed the data. S.Y. and M.K. performed theoretical calculations. S.H., C.K., and C.A. provided in-depth discussion of the project. S.Y., M.K., and Y.L. wrote the manuscript.

\section{Additional information}

Supplementary Information accompanies this paper at https://doi.org/10.1038/s41467018-04689-5.
Competing interests: The authors declare no competing interests.

Reprints and permission information is available online at http://npg.nature.com/ reprintsandpermissions/

Publisher's note: Springer Nature remains neutral with regard to jurisdictional claims in published maps and institutional affiliations.

(c) (i) Open Access This article is licensed under a Creative Commons Attribution 4.0 International License, which permits use, sharing, adaptation, distribution and reproduction in any medium or format, as long as you give appropriate credit to the original author(s) and the source, provide a link to the Creative Commons license, and indicate if changes were made. The images or other third party material in this article are included in the article's Creative Commons license, unles indicated otherwise in a credit line to the material. If material is not included in the article's Creative Commons license and your intended use is not permitted by statutory regulation or exceeds the permitted use, you will need to obtain permission directly from the copyright holder. To view a copy of this license, visit http://creativecommons.org/ licenses/by/4.0/.

(C) The Author(s) 2018 